\title{
Limb reconstruction surgery in China: an evaluation of its role in the largest developing nation
}

\author{
Lei Huang $\cdot$ Liang He $\cdot$ Jie Wei $\cdot$ Manyi Wang
}

Received: 28 August 2007/ Accepted: 18 October 2007/Published online: 14 November 2007

(C) Springer-Verlag 2007

\begin{abstract}
China is the "economic tiger" of the twentyfirst century. Its new economic policies have overturned the outlook and futures of many industries in this nation, and parallel to this industrial progress have been strides in medicine. However a country steeped in a history of several millennia does not replace generations of experience nor knowledge in a few decades. The author explores the history of limb reconstruction surgery in this country, setting it in context of a population exposed to both traditional Chinese medicine and Western medicine.
\end{abstract}

Keywords External fixation - Ilizarov ·

Limb reconstruction $\cdot$ China

\section{Introduction}

Limb reconstruction surgery is a subspecialty of orthopaedics which aims to restore the anatomical and functional integrity of a limb without resort to amputation or prosthetic replacement. Although many would attribute the beginnings of modern limb reconstruction surgery to the work by Ilizarov, today it embraces more than just this method but also microsurgery techniques as well as established AO principles.

Limb reconstruction surgery was first started in P. R .China by Li Qihong in 1982 with his half-ring sulcated external fixator $[1,2]$. Ilizarov's methods and device were

\footnotetext{
L. Huang $(\bowtie) \cdot$ L. He $\cdot$ J. Wei $\cdot$ M. Wang

Department of Orthopaedics and Traumatology, Beijing Ji shui Tan Hospital, The fourth Medical College of Peking University, No.31, Xin Jie Kou Dong Jie, Xicheng district, Beijing 100035, People's Republic of China

e-mail: huangleibear@yahoo.com.cn
}

not popular in China until late of 1980's as it was thought demanding and time consuming, and the fixator (whether imported or copied components) were difficult to obtain at that time [3] (Fig. 1).

\section{A need for limb reconstruction}

Rapid growth and modernisation in China has led to a tremendous increase in motor vehicles occupying the same road space as the millions of pedestrians and cyclists. Consequently injuries and mortality from road traffic accidents are increasing year by year. The total number of accidents in 2003 were 667,507; of deaths there were 104,372 and of injuries 494,174. This represented a 6.22 , 5.47 and 6.38 -fold increase, respectively over the figures for 1978 [4, 5]. For example, the incidence of orthopaedic trauma in Shenzhen (a city of south China's Guangdong province-China's first special economic zone) increased from 126.46/100,000 in 1994 to 308.55/100,000 in 2003. Most patients were between 10 and 40 years old; $80 \%$ were male. As for location, $76.86 \%$ of the cases occurred in its two industrial districts. Most were treated in hospitals of district level or below rather than in tertiary level hospitals despite many of the injuries being high energy limb trauma or multiple fractures [6]. The majority paid their medical bills by themselves even though they had limited financial resources.

The spectrum of trauma and complications arising thereafter has been such that more patients with bone defects, posttraumatic nonunion and malunion, and osteomyelitis present to surgeons with general Orthopaedic experience. The subspecialisation needed to treat these difficult problems is now recognised and increasing numbers of surgeons are attracted to train in this field. 


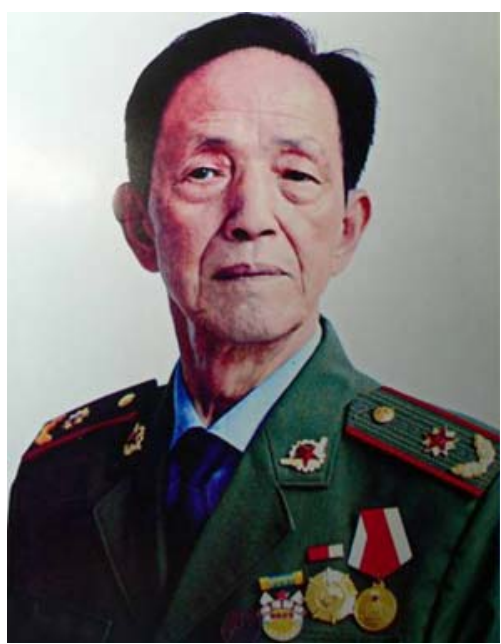

Fig. 1 Li QiHong, Father of limb reconstruction in China

\section{Western orthopaedic training in China}

Modern orthopaedic education began at the Peking Union Medical college (PUMC) in 1921. Founded by the Rockefeller Foundation, the hospital's first orthopaedic department was organized by Dr. George W. Van Gorder from Massachusetts General Hospital, USA. Its first Chinese Orthopaedic Chief at the PUMC was Meng Chimao (1897-1980), a Chicago Rush Medical graduate who undertook postgraduate training under Arthur Steindler and M.N. Smith-Peterssen. He co-authored "Primer on Fracture and Dislocation" with Leo J. Miltner, the first book of its kind in China. He became the director of Beijing Ji Shui Tan (JST) Hospital, a government-run general and teaching hospital of Peking University, built in 1956 and incorporating an Orthopaedic and Traumatology Department of 300 beds and an Orthopaedic Research Institute. The number of beds has now increased to 1,050 with all major subspecialties covered, 512 of them for orthopeadics (Fig. 2).

In parallel to the exposure to Western orthopaedics, China was instrumental in the field of hand microsurgery and replantation. Dr. Wang Shuhuan (an academician of the Chinese Academy of Engineering) founded China's first hand surgery department in Beijing JST hospital in 1958. He succeeded in re-anastomosing blood vessels in a rabbit ear under microscopy in 1963, and replantation of a rabbit ear and replantation of fingers in 1964 [7, 8]. In the same year a successful replantation of a hand, arm and ankle were completed by his colleagues. The first Chinese book on hand surgery was written on the basis of clinical experience of Beijing JST hospital's department of hand surgery, published in 1978, Wang Shuhuan was the chief editor.

Further expansion in training has been possible by the involvement of Chinese surgeons in foreign training programmes and international meetings since the early 1980's.

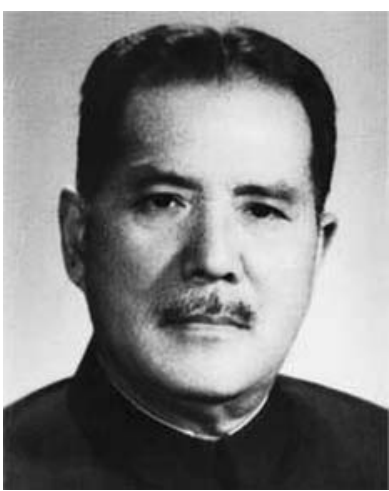

Fig. 2 Meng JiMao, Author of "Primer on Fracture and Dislocation"

This is in addition to lectures and technique demonstrations by overseas surgeons in China itself. The increase in familiarity with modern and western orthopaedic procedures has brought many top orthopaedic equipment manufacturers to this country, thus making it possible for surgeons here to keep up-to-date (Fig. 3).

The reputation and orthopaedic landmarks made by the Beijing JST hospital attract 50-100 Orthopaedic surgeons (mostly at attending level) from across China each year. Several orthopaedic surgeons from this unit are frequent members of faculty as national and international meetings. This precedence is associated with a high percentage of tertiary referrals to the Beijing JST hospital (Fig. 4).

\section{Introducing limb reconstruction in China}

The orthopaedic techniques of microsurgical reconstruction, Ilizarov distraction histogenesis and the more familiar

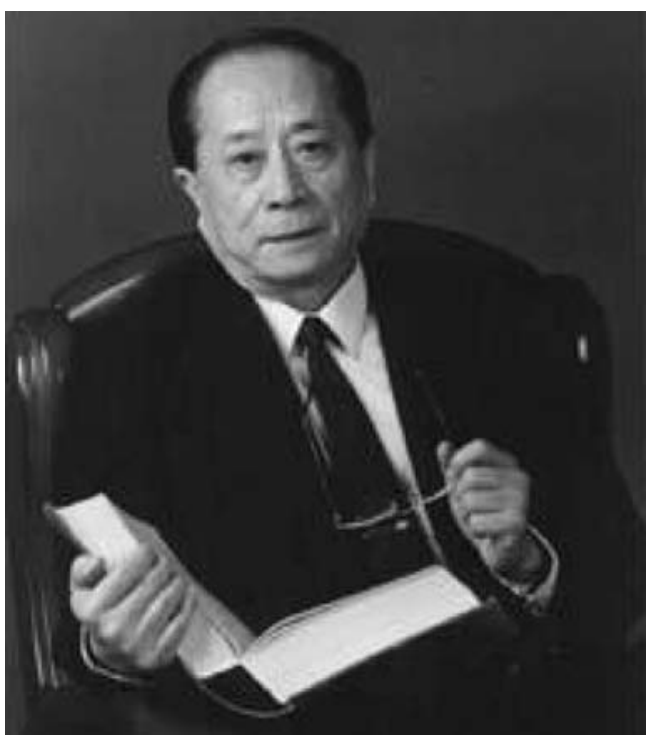

Fig. 3 Wang ShuHuan, Microsurgery pioneer 
Fig. 4 The Beijing JST

Hospital in Beijing

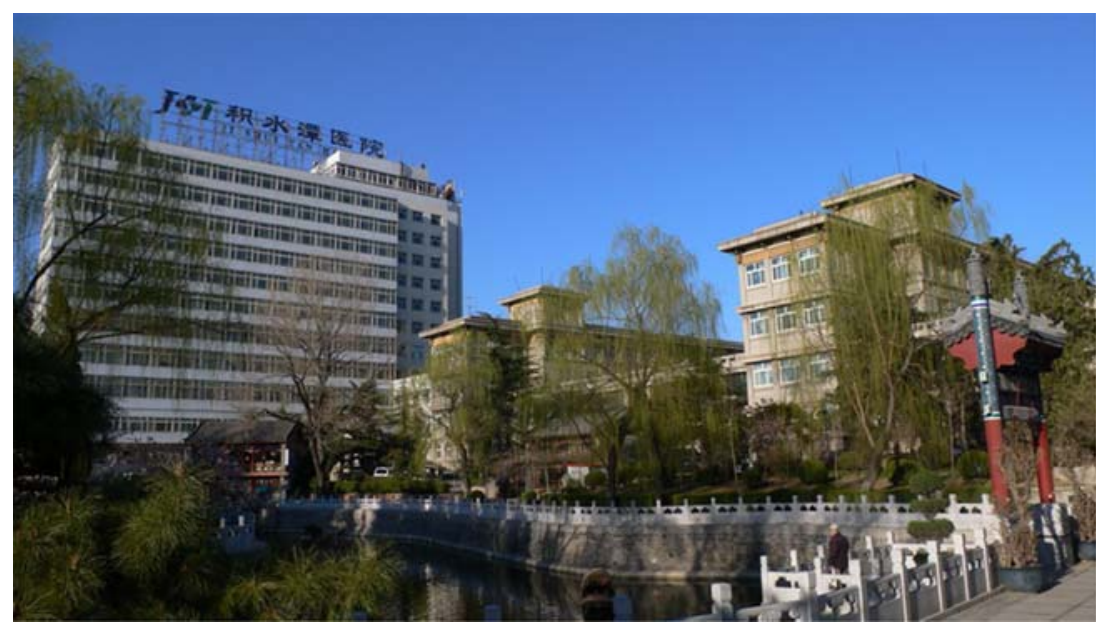

AO principles amalgamate as working tools for limb reconstruction. These techniques have been in use at the JST for some decades, but until recently not drawn together for the purpose of solving problem fractures, non-union and osteomyelitis. With reference to reconstruction using external fixators, there is greater familiarity with circular than monolateral devices. In many respects, the process of building a unit capable for limb reconstruction involves recruitment, training, and experience. Residents and an attending doctor in the unit work with a senior consultant doctor for 6 months. Through lectures, demonstrations and discussions on technique and rationale of different fixator constructs, visiting doctors and nurses become familiar with the surgery and various devices. Currently two staff surgeons are sufficiently trained and experienced to independently deal with cases needing bone reconstruction.

Limb reconstruction is still relatively new in China. The problems are the same as elsewhere which, in the main, are a treatment programme which is demanding, costly and lengthy. Even so, cases of orthopaedic trauma increase with the economic development in China yearly. Most of the victims are young male workers or farmers and road traffic accidents are the primary cause. Medical bills are met through self-pay even though patients have limited financial resources. China's vast size (east to west is $5,200 \mathrm{~km}$ and south to north is $5,500 \mathrm{~km}$ ) influences the ability of patients to travel to the well-equipped hospitals in Beijing, Shanghai and other big cities.

Epidemiological papers on orthopaedic trauma has provided evidence to the government for greater attention to this area and encouraged measures for control and prevention strategies. There is need for education to reach both doctors and patients such that an awareness of these new techniques may facilitate appropriate case referrals. Whilst amputation is an alternative and rehabilitation facilities make it a realistic option in more developed nations, it is a difficult primary treatment recommendation in China. Primary amputation is still viewed as likely to lead to loss of gainful employment and associated with a sense of hopelessness as most patients are the bread-winners.

With a relatively new surgical technique as limb reconstruction is for China, it is important to explain the treatment plan in detail to a patient before surgery. Advantages and disadvantages of amputation and reconstruction must be addressed, even though the former is rarely accepted readily. Some adjustment to treatment strategy is needed according to the patient's ability to meet the medical bill; whilst this is not prevalent in developed nations, it is a fact of medical practice in most developing countries. For example, if there is a bone defect of less than $4 \mathrm{~cm}$ and the cost of treatment is met entirely by the patient, I usually advocate open bone grafting as the surgical strategy as it costs much less than bone transport. If bone transport or other similar reconstructive technique is proposed, an open discussion of the complications and risks is undertaken together with education of ways to avoid common problems-pin site care, distraction regimes, and exercise. Patients are provided contact telephone numbers or e-mail addresses for support during treatment. Though bone reconstruction is lengthy and costly, an attempt to shorten the morbidity time and decrease the number of operations can be achieved by careful preoperative plans and choice of strategy. Examples are using the acute shortening-lengthening technique, covering wound defects with split-thickness skin grafts at the time of debridement, and addressing the docking site of a bone transport early.

\section{Workload at the limb reconstruction unit of the Beijing JST hospital}

There are 1,050 beds in the JST hospital in Beijing: 512 of the total beds are for orthopaedics and it covers all the major subspecialties - the hand, burns, bone tumour and trauma units are well known in China [9]. Over 20,000 
Fig. 5 The author with patients undergoing limb reconstruction and their wives

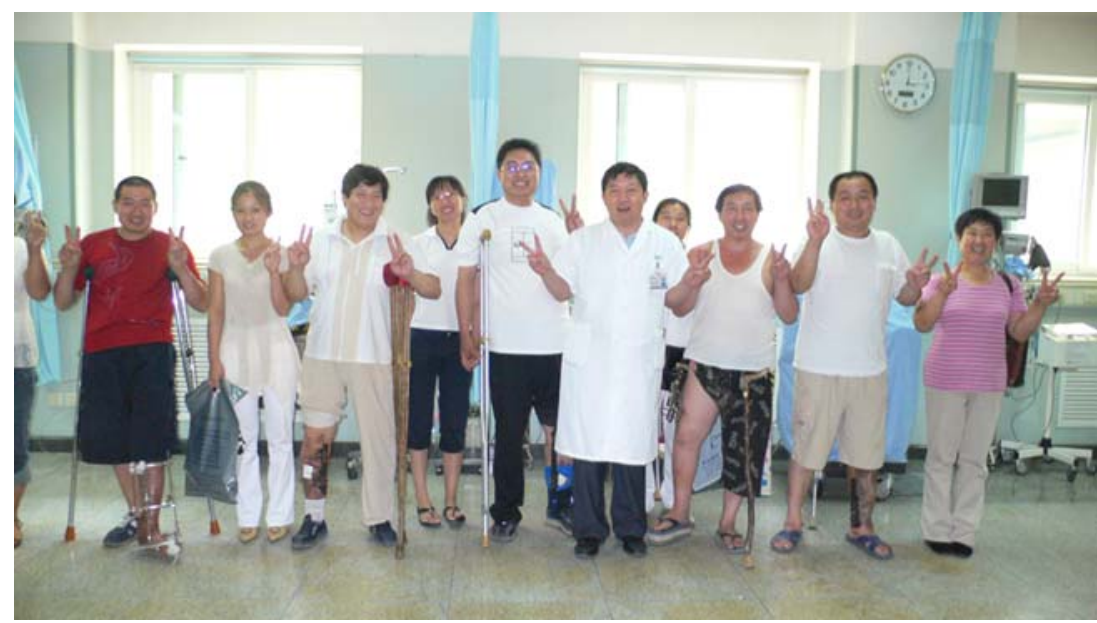

orthopaedic operations were completed in 2005-the largest number of such procedures in 1 year in a single hospital in China [10].

The limb reconstruction unit at the Beijing JST hospital receives $78 \%$ of its referrals from west China. The open cancellous bone grafting technique of Pappineau was used regularly for bone defects less than $4 \mathrm{~cm}$ (the longest was $6.5 \mathrm{~cm})[11,12]$. In 1994 ring fixators were used to manage bone defects over $4 \mathrm{~cm}$. Ring fixators were used more frequently in the initial stages as local designs and devices were available. However access to monolateral fixators designed specifically for limb reconstruction has improved; between July 2001 and March 200682 cases of bone defect or limb length discrepancy were successfully treated using a monolateral external fixation system. Sixtyfour of the cases were of tibial bone defects or a length discrepancy with an average of $9 \mathrm{~cm}(5-22 \mathrm{~cm})$; there were seventeen cases of monolateral fixator use in the femur with an average defect of $7 \mathrm{~cm}(4-13 \mathrm{~cm})$. There was a single case of humeral defect reconstruction of $7 \mathrm{~cm}$ [13, 14] (Fig. 5).

\section{Interactions of traditional Chinese Medicine (TCM) and Western Medicine (WM)}

Orthopaedics in China has roots in both TCM and WM [15]. TCM dates back to over 3,000 years: Hua Tuo (200 $\mathrm{AD})$ was a pioneer of oral anaesthesia and wound excision. Treatment of fractures, dislocations and deformities of bones and joints was described by a number of authors since Tang Dynasty (618-907AD). In several classic medical texts from the era, fracture reduction technique was emphasized as needing eight steps of which the most notable one was bending of the tips of fracture fragments, i.e. sliding one end on the other in planes perpendicular to each other and then levering for reduction. This was followed by shaking, pounding and "rearrangement" of soft tissue. Fracture fixation with short splints was also developed. Improvement of local circulation and dissipation of swelling by oral intake and local application of herbal remedies was advised [16].

Western medicine in China began as early as in the Ming Dynasty (1368-1644) through Roman Catholic missionaries and merchants. After the Opium War of 1840-1842, it became widely spread and a number of hospitals and medical schools were established. In 1887 Keer established the China Medical Missionary Journal, a predecessor of the English edition of the Chinese Medical Journal, which has witnessed the progress of orthopaedics in China.

The argument of abolishing TCM since Western scientific concepts were introduced to China has been going for over 100 years. The pattern of judging TCM from the perspective of Western criteria still exists. According to those who advocate abolishing TCM, WM is based upon established sciences of physics, chemistry, biology and statistics. The curative effects and side effects of WM can be explained and proven by its theories and clinical experiments. In contrast, the Yin and Yang and Five element theories of TCM seem to be too abstract, metaphysical and difficult to explain through science. Therefore TCM is often viewed as no more than an accumulation of experience and not a science, even though it has some effective medicine and therapies. Some concepts and methodologies were ahead of its time, the "eco-medicine" concept is an example. There also have been spectacular advances in the management of especially viral infections and chronic diseases.

However it is a fact TCM has developed its own rules and a system based on deep roots of the Chinese culture. These roots have given a system of medicine that has benefited the Chinese people for millennia. TCM is more than just a technology as it involves a philosophy of 
preserving health and balance, in keeping with the way the Chinese perceive things. To many Chinese, there is no reason to disregard this traditional medical legacy.

Thus whilst TCM and WM are two different systems, they share the same objective - human health. In the early 1950 's, integration of TCM and WM was advocated. The first breakthrough in orthopaedics was in the treatment of fracture of both forearm bones; in this setting, TCM doctors lay much emphasis on separating the bones for reduction. Later anatomical studies confirmed this technique as important in achieving reduction. Whilst fracture stabilisation was through short, thin wooden splints, these did not extend beyond the joints, thereby allow patients to move the elbow and wrist. Fractures so treated healed quickly and non-union is practically unknown to TCM practitioners. These results were perhaps not as good radiologically when compared to open surgery, the functional results are surprisingly satisfactory [17].

Both TCM and WM have merits and disadvantages. Clinical experience has shown that no single method of treatment is satisfactory for all types of fractures, and TCM is not advisable for open or multiple fractures, or for articular fractures. There are also other factors which may decide treatment choice: economic pressures may persuade urban area workers in China to opt for open surgery as this is sometimes seen as a faster route to return to work.

\section{Funding health care in China}

China is the largest developing nation. There is a low level of urbanisation in the country despite the presence of major international cities and a large income gap between urban and rural, largely agriculturally-based, residents. The Chinese government has been trying to establish a health care system that guarantees every citizen basic medical services and whilst many examples of nationalised health care systems exist internationally none seem suitable for China.

Three decades ago urban residents enjoyed state-funded hospital services, and the rural population had access to subsidized clinics run by "barefoot doctors" that were mainly middle school graduates trained in first aid. This service, essentially free, helped almost double the country's average life expectancy from 35 in 1949 to 68 in 1978. However, the medicare system created then only covered government and State-owned enterprise employees and was unfair. When China began economic reforms in the early 1980s, the old system was dismantled as the nation attempted to switch to a market-oriented health system. As early as 1997, a public healthcare insurance plan was launched in the cities. But this has turned out not to solve the problem. China's Ministry of Health figures indicate that almost $90 \%$ of the rural population today has no health insurance. In 1975 , by contrast, about $85 \%$ of rural residents had community-financed health care [18]. The urban figure is not much better at $60 \%$, yet two-thirds of China's government funding for medicare is spent on urban areas which are home to only one-third of the population. A report by the Chinese Academy of Sciences says $80 \%$ of those funds to urban areas is used by only 8.5 million people in a country whose population exceeds one billion [19].

It is apparent the current system is unsustainable. By most measures, China has prospered enormously from its shift to economic liberalization. The problem of funding a health care system is one of the challenges the mainland is encountering; numerous other countries have wrestled with the same basic questions that China's mainland faces today: what is the best system for delivering health care, and who should pay for it? To its credit, the Chinese government has made clear that it intends to overhaul the country's health care approach to better serve its citizens [18].

\section{The future of limb reconstruction in China}

Whilst there is clearly a need for this type of specialist surgery, the dilemma between expensive treatment and the issues of funding basic medical care to the whole population arises. A 2006 national survey showed $48.9 \%$ of Chinese did not bother or could not afford to see a doctor when they fell ill, and $29.6 \%$ could not be admitted to a hospital on medical advice because the cost was too high [20].

Whilst a solution is awaited (and this may be elusive as judged by the problems that continue in many more developed countries where a national healthcare system remains), the unit at the JST hospital has adopted several strategies in order to continue delivery of this specialist treatment and keep costs to a minimum. Several strategies are implemented:

Patients purchase only half pins or wires but rent the relevant fixator components from the supplier of medical equipment

Liaison between the commercial external fixator suppliers and the hospital can, in certain circumstances, allow the use of fixator components rent-free, and the half pins obtained as second-hand purchases. Re-use of components and half-pins is commonplace in developing nations and the quality of original equipment testifies to re-use, but the permission to proceed under these circumstances is taken with approval from patient and government authority.

Local manufactured (often design-similar devices) fixators are available at reduced cost

With such adjustments, specialist treatment can reach more patients. About $80 \%$ of patients managed with 
original equipment manufacturer fixators were paid for by the Chinese government, their employers or insurance companies. Further expansion of this specialty is possible if there is collaboration between the commercial enterprises which manufacture and distribute the original external fixators and developing nations as China. Much of the reluctance of patients to choose OEM fixators lie with cost-if these implants or fixators were made by joint ventures locally, purchase costs can be decreased considerably; coupled to an approved rental scheme this can lead to a exponential increase in uptake of this much needed surgical technique. It is entirely feasible that such joint collaboration can reduce the gulf in prices between OEM devices and locally made external fixators to a degree that it would seem churlish not to choose the higher quality product.

\section{References}

1. Li QH, Ou B, Zhou Z (1985) Distraction epiphisiolysis for limb lengthening: a preliminary report of 55 cases (In Chinese). Chin J Orthop 23(2):106-109

2. Li QH, Zeng X, Ou B (1984) A half-ring sulcated external fixator: a new design and its clinical application (in Chinese). Chin J Orthop 6:348-350

3. Feng $\mathrm{CH}$ (2004) Historical materials of modern orthopaedics in China (In Chinese). University Medical Press, Beijing, pp 100

4. Traffic Administration Bureau (2004) Statistics of road traffic crashes of People' s Republic of China (in Chinese), Beijing

5. Traffic Administration Bureau (1988-2003) Statistics of road traffic crashes of People' s Republic of China (in Chinese), Beijing
6. Jiang H, Xiao D, Li W (2006) A survey of orthopaedic trauma cases from 1994 to 2003 in Shengzhen (in Chinese). Chin J Orthop 8(12)

7. Lu JZ, Wang SH (1965) Preliminary experience of reanastomosis of finger artery (in Chinese). Chin J Surg 13(2):179-180

8. Lu JZ, Wang SH (1963) Blood vessel re-anastomosis in a rabbit ear (in Chinese). Beijing Medicine 1(1):20-22

9. Feng CH (2004) Historical materials of modern orthopaedics in China (in Chinese). Beijing, pp 420

10. Tian W (2006) Address of the President of the hospital (in Chinese). Traumatol Orthop Q 35(1)

11. Huang L, Li B, Liu Y (2005) Open bone grafting as the treatment for the infected fracture nonunion (in Chinese). Chin J Orthop 25(1):30-34

12. Huang L, Liu Y (1998) One-stage open cancellous bone grafting of infected fracture and non-union. J Orthop Sci 3(6):318-323

13. Huang L, Zhu F, Wang S (2006) Femoral lengthening or transport in combination with an intramedullary nail (in Chinese). Chin J Orthop Traumatol 2006(8):634-638

14. Huang L, Xie M, Wang J (2004) Management of bone defects with orthofix limb reconstruction system (in Chinese). Chin J Orthop Traumatol 6(10):1096-1101

15. Feng $\mathrm{CH}, \mathrm{Yu} \mathrm{D}$ (1992) The modern historic background and development of orthopaedics in China (in Chinese). Chin J Med Hist 22:193

16. Shang TY, Gu Y, Huang Q (1995) Chinese osteosynthesis (in Chinese). Tianjin Science and Technology, Tianjin

17. Fang H-C, Ku Y-W, Shang T-Y (1963) The integration of modern and traditional Chinese medicine in the treatment of fractures: a simple method of treatment for fractures of the shafts of both forearm bones. Chin Med J 82:493

18. Go R (2006) Mainland's health care system: Searching for a cure. China Daily HK edn, 2nd September

19. Rong J (2006) State cure needed for medical ills. China Daily, 17 th October

20. Wu J (2007) Social problems and how to solve them. China Daily, 6th March:10 